\title{
Frequency spectra for fluids beyond the mean spherical approximation
}

Cite as: J. Chem. Phys. 101, 4083 (1994); https://doi.org/10.1063/1.467525

Submitted: 28 February 1994 . Accepted: 16 May 1994 . Published Online: 31 August 1998

Johan S. Ho/ye, and Enrique Lomba
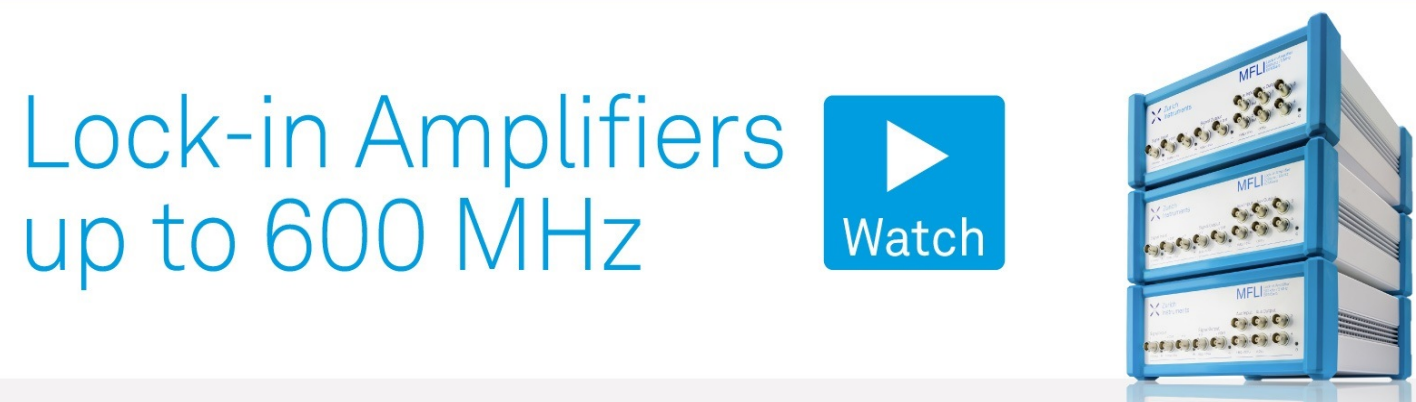

J. Chem. Phys. 101, 4083 (1994); https://doi.org/10.1063/1.467525 


\title{
Frequency spectra for fluids beyond the mean spherical approximation
}

\author{
Johan S. Høye \\ Gruppe for Teoretisk Fysikk, Universitetet i Trondheim-NTH, N-7034 Trondheim, Norway \\ Enrique Lomba \\ Instituto de Química Física Rocasolano, CSIC, Serrano 119, E-28006 Madrid, Spain
}

(Received 28 February 1994; accepted 16 May 1994)

\begin{abstract}
In this paper, we investigate the frequency spectra of fluids (or fluid-like disordered materials) composed of particles with embedded harmonic oscillators. The general treatment is particularized at the low density limit, and a working scheme to add corrections to the mean spherical approximation for arbitrary density is introduced. A detailed comparison with an exactly solvable model in one dimension is also presented.
\end{abstract}

\section{INTRODUCTION}

It has been known for a decade that dynamic properties of fluids can be obtained from classical statistical mechanics of fluids at equilibrium. This became apparent when the quantized fluid of polarizable particles was solved independently by Høye and Stell ${ }^{1}$ and by Thompson, Schweitzer, and Chandler ${ }^{2}$ in the mean spherical approximation (MSA). The quantization was restricted to the fluctuating dipole moments. In the model with fluctuating dipole moments introduced and solved by Pratt $^{3}$ and by Hoye and Stell ${ }^{4}$ in the MSA for the classical case, one assumes that each particle has a dipole moment that fluctuates in a harmonic oscillator potential. Between dipole moments in different particles, one then has the usual dipole-dipole interaction. The abovementioned solution of the quantum-mechanical problem was performed by utilizing the path integral formalism described by Feymann and Hibbs. ${ }^{5}$ Using this formalism, it was noted that the quantum problem was equivalent to a classical polymer problem (i.e., polymer rings of length $\beta$ in the direction of imaginary time dimension). Then this polymer problem can be solved in the MSA.

By solving the quantum problem, it became clear that dynamical information was also at hand. Thus frequency spectra of disordered systems of coupled harmonic oscillators could be obtained and were found by Høye and Olaussen ${ }^{6}$ and Chandler, Schweitzer, and Wolynes. ${ }^{7}$ Later, several similar disordered or fluid-like systems have been investigated, and the method has been extended to the determination of energy bands formed in these systems due to coupling of atomic orbitals. ${ }^{8,9}$ This generalizes the wellknown formation of energy bands in regular crystals. The problem of atomic orbitals with couplings can be related to a harmonic oscillator problem that can be represented by the same type of matrices whose eigenvalues are sought for. Clearly, the matrix problem can be solved for each frequency or eigenvalue separately. In this respect, the quantum problem somehow is removed. This should not be surprising as harmonic oscillator eigenfrequencies do not depend on quantization. The matrix problem can now be represented by the classical partition function of coupled harmonic oscillators for each frequency separately. ${ }^{10}$ Although this points out more clearly that different frequencies can be treated inde- pendently, it represents nothing but the original quantum path integral partition function if one restricts it to a quenched situation, i.e., the positions of particles are fixed in space. Fourier transformation in imaginary time as performed by the MSA then splits the latter problem in independent imaginary frequencies. Nevertheless, it should be noted that this decoupling will disregard the effect of induced dispersion forces upon spatial correlations. In principle, the decoupled problems could also be treated as annealed systems too. However, the latter will not make sense since different frequencies would then yield different equilibrium configurations. This is not possible for one given system in a specific thermodynamic state. In the MSA, this was not at issue, but by looking for corrections to the MSA, this distinction becomes relevant.

The notion that each frequency can be regarded separately as an equilibrium problem was also used by Hoye and Stell in their approach to the MSA solution of the refractive index of a polarizable fluid. ${ }^{11}$ Høye and Lomba performed numerical evaluations in this context, and computed complex dielectric constants and time dependent correlation functions. ${ }^{12}$

Later works that go beyond the MSA have been performed. Such approaches may be denoted as nonlinear theories, since the corresponding direct correlation function will no longer be just linear in the interaction outside the hard cores. In this context, Chen and Stratt have employed the EXP approximation. ${ }^{10}$ They consider continuum fluids and obtain improved results in the low density limit. One must attribute this improvement to the fact that the EXP approximation appropriately incorporates the second virial coefficient (disregarding quenched positions). Compared with computer simulations, ${ }^{13}$ the frequency spectrum then has a more correct form with a kink at the single oscillator frequency, whereas the MSA results have this feature smoothed out. The kink, we think, is the result of taking into account the eigenfrequencies of a pair of particles (coupled oscillators) at different separations. At large separations, the frequencies will then cluster around the single oscillator frequency giving the sharp maximum in the spectrum. This is verified by the low density analysis performed by Gibbons et al. ${ }^{14}$ and Bush et al. ${ }^{15}$

Recently, Wan and Stratt have also performed graph ex- 
pansions and obtained improved results for instantaneous normal modes of liquids. ${ }^{16}$ In the present work, we will continue along this line to obtain a correction to the MSA in a systematic approximation.

The rest of the paper can be sketched as follows: In Sec. II, we introduce our theoretical treatment. Its low density limit is considered in Sec. III, and corrections for arbitrary density are taken into account in Sec. IV. Then, Sec. V is devoted to a detailed comparison with a exactly solvable case of a one-dimensional lattice system. Finally, some remarks concerning the general treatment of the continuum case can be found in Sec. VI.

\section{THEORY}

Our system consists of particles that form a fluid (or fluid-like disordered system). The particles contain embedded harmonic oscillators that for instance may represent fluctuating dipole moments. For simplicity, one single oscillator is assumed to be present in each particle, but several may be included without changing the formalism. Between each pair of oscillators, there is a pair interaction of the form

$$
\phi_{s_{1} s_{2}}(r)=s_{1} s_{2} \phi(r)
$$

where $s_{i}(i=1,2)$ are the coordinates of the internal motions of the oscillators and $r$ is their spatial separation. Each single oscillator has an eigenfrequency $\omega_{0}$ and is tied to its center by a force $M \omega_{0}^{2}$, where $M$ is the reduced mass. In general, the coordinates $s_{i}$ can be vectors $s_{i}$, and $\phi(r)$ can be a matrix.

To obtain our results, we use the $\gamma$ ordering as a basis to select graphs to perturb the MSA. $y$ is the inverse range of the perturbing interaction and can be used as a perturbing parameter. ${ }^{17}$ The reference system is then usually hard spheres in the continuum case or forbidden multiple occupancy in the lattice gas (or spin system) case. In the mean field limit $\gamma \rightarrow 0$, well-established results such as the van der Waals equation of state are obtained. The MSA turns out to be the same as the first $\gamma$-ordered correction to mean field theory, where the perturbing potential inside the hard core is chosen such that the exact core condition on the pair correlation function is preserved.

To consider higher orders in $\gamma$, we utilize the expressions found by $\mathrm{H}$ bye and Olaussen in the investigation of the twodimensional Coulomb gas. ${ }^{18}$ Their investigation verified the results obtained by Kosterlitz and Thouless on the special kind of phase transition that occurs in this system. ${ }^{19}$

A special feature of terms dealt with in Ref. 18 is that they can be identified with chemical association, i.e., ions bind into neutral dipolar pairs due to the strong ionic forces, and an equation of chemical equilibrium appears. In the present instance, this turns out to be a convenient feature too, although pairing when compared to ionic systems should be of less importance. Besides, the second virial coefficient will be included exactly. Its inclusion would have given rise to a divergence had it not been for the resummation that yields chemical association. The reason for divergence lies in the interaction (2.1) that is unbounded since $s_{i}$ is not limited either. Then its exponential can always outweigh the constant $\times s^{2}$ term in the chemical potential. The latter term is the binding potential of each oscillator.

In order to deal with the oscillator configurations, we, as usual, employ the trick of regarding different oscillator states to represent different species of a mixture, as originally introduced by Onsager..$^{20}$ The expressions of Ref. 18, which beyond the MSA only are valid at low density, will be established below. Later we will make modifications to extend them to arbitrary density.

In general, the chain bond can be defined such that its Fourier transform $\tilde{\mathscr{E}}(k)$ in terms of hypervertices $\tilde{\mu}(k)$ and potential bonds $\tilde{v}(k)$ can be written

$$
\tilde{\mathscr{E}}(k)=\frac{\tilde{v}(k)}{1-\tilde{\mu}(k) \tilde{v}(k)},
$$

where for a mixture, these quantities are matrices with elements $\tilde{\mathscr{C}}_{i j}(k)$, etc. The potential bond $\tilde{v}(k)$ in terms of the interaction (outside the hard cores) is given by

$$
v_{i j}(r) \rightarrow-\beta \phi_{i j}(r) \rightarrow-\phi_{s_{1 K} s^{2 K}}(r) \rightarrow-\phi_{s_{1} s_{2}}(r) .
$$

$s_{i K} \rightarrow s_{i}(i=1,2)$ are here the Fourier transform variables of $s_{i_{p}}$ along the polymer chains, such that the $\beta$ factor drops out. ( $p$ denotes the discretized position along the chain.) This is balanced by a similar compensation in Eq. (2.11) below.

The hypervertex $\tilde{\mu}(k)$ contains first of all the simple $\rho$ vertex which in cases such as the present one, where the average interaction is zero, will yield simple chain bonds that can be used in higher order graphs, but $\tilde{\mu}$ can also be extended to contain all allowed graphs, such that the full pair correlation function $h(r)$ can be written (in matrix notation) as

$\rho+\rho^{2} \tilde{h}(k)=\frac{\tilde{\mu}(k)}{1-\tilde{\mu}(k) \tilde{v}(k)}=\tilde{\mu}(k)+\tilde{\mu}(k) \tilde{\mathscr{E}}(k) \tilde{\mu}(k)$.

In Ref. 18 , attention is focused on $\tilde{\mu}(k)$, and it is approximated by

$$
\mu_{i j}(r)=\rho_{0 i} \delta_{i j}+\rho_{i} \rho_{j} f_{i j}(r)
$$

with

$$
f_{i j}(r)=\exp \left(\mathscr{E}_{i j}\right)-\mathscr{C}_{i j}-1 \quad\left[\mathscr{C}_{i j}=\mathscr{C}_{i j}(r)\right]
$$

Here $\rho_{0 i}$ denotes the total density, while $\rho_{i}$ denotes the density of free particles of species $i$ not bound into pairs. By the $\gamma$ ordering, the lowest power when expanding $f_{i j}(r), C_{i j}^{3}$ represents the next order beyond MSA. Higher powers in $\mathscr{E}_{i j}$ are then included to account for the second virial coefficient correctly. In the case of ionic systems, where forces are strong, this is important for the association into pairs. Now there will be an equation of chemical equilibrium

$$
\rho_{0 i}=\rho_{i}+\sum_{j} \rho_{i} \rho_{j} K_{i j}
$$

with the constant of chemical equilibrium

$$
K_{i j}=\int\left[\exp \left(\mathscr{C}_{i j}\right)-\frac{1}{2} \mathscr{E}_{i j}^{2}-\mathscr{C}_{i j}-1\right] d \mathbf{r}
$$


[Note that $f_{i j}(r)$ is the derivative of the integrand above with respect to $\mathscr{C}_{i j}$.] The internal energy (not including kinetic energy) $U$ per volume unit is now obtained via the pair correlation function (2.4). Using Fourier transformed quantities and inserting Eq. (2.5), one obtains

$$
\begin{aligned}
-\beta U= & \frac{1}{2} \frac{1}{(2 \pi)^{d}} \operatorname{Tr} \int \frac{\tilde{\mu}(k) \tilde{v}(k)}{1-\tilde{\mu}(k) \tilde{v}(k)}-\frac{1}{2} \sum \rho_{0 i} v_{i i}(0) \\
= & \frac{1}{2} \sum \rho_{0 i}\left[\mathscr{C}_{i i}(0)-v_{i i}(0)\right] \\
& +\frac{1}{2} \sum_{i j} \rho_{i} \rho_{j} \int f_{i j}(r) \mathscr{E}_{i j}(r) d \mathbf{r} .
\end{aligned}
$$

Integration of this with respect to $\beta$ can be performed analytically to obtain the Helmholtz free energy utilizing the relations above. ${ }^{18}$ This quantity nonetheless will not be needed here and we omit its derivation. Differentiation of the free energy with respect to $\rho_{0 i}$ then yields the chemical potential $\mu_{i}$ as

$$
\beta \mu_{i}=\log \rho_{i}-\frac{1}{2}\left[\mathscr{C}_{i i}(0)-v_{i i}(0)\right] .
$$

Note that here $\rho_{i}$ not $\rho_{0 i}$ occurs in the ideal gas logarithmic term.

By application of the above expressions to the embedded harmonic oscillators, the subscripts $i$ and $j$ will be the harmonic oscillator coordinates $s_{i}(i=1,2)$. The $s$ dependence of the chemical potential is now determined by the harmonic oscillator potential at zero frequency plus its modification at nonzero frequency. Thus in accordance with the path integral method, we have ${ }^{1}$

$$
\beta \mu_{s}=-\frac{1}{2} \sigma\left(K^{2}+\hbar^{2} \omega_{0}^{2}\right) s^{2}+\text { constant }
$$

with $\sigma=M / \hbar^{2}$, and $K$ is the imaginary frequency, such that $K^{2}=-\hbar^{2} \omega^{2}$, and it is furthermore the Fourier transform variable in imaginary time along the periodic polymer chain of length $\beta$. Expression (2.10) inserted in Eq. (2.9) then yields for the density distribution of "free" particles

$$
\rho_{s}=B \exp \left(-\frac{1}{2} a s^{2}\right),
$$

where $B$ is some constant, while

$$
a=\sigma\left(K^{2}+\hbar^{2} \omega_{0}^{2}\right)-(\mathscr{C}(0)-v(0)) .
$$

There is no $\beta$ factor in $a$ in accordance with the comment below Eq. (2.3). $\mathscr{E}(0)$ and $v(0)$ follow from the correspondences

$$
v_{i j}(r) \rightarrow v(r) s_{1} s_{2}, \quad \mathscr{G}_{i j}(r) \rightarrow \mathscr{B}(r) s_{1} s_{2},
$$

where the form of $\mathscr{E}_{i j}(r)$ follows from the form of $v_{i j}(r)$ via Eq. (2.2). Here one can note that the use of $\rho_{s}$ instead of $\rho_{0 s}$ has the effect that the explicit appearance of the second virial coefficient for $\mu_{s}$ does not show up in the exponent of Eq. (2.11). [Instead it comes forth via $K_{i j}$ of Eqs. (2.6) and (2.7).] Its appearance would have resulted in a divergence, as mentioned earlier, due to the interaction (2.1). This divergence is present in the treatment of Ref. 10, but expanding it off the exponent in Eq. (2.11) will tame it such that it does not outweigh the $s^{2}$ term for large $s$.
By use of Eq. (2.8), we can now establish an expression for the internal energy from the perturbing interactions. We get for each value of $K$,

$$
\beta U_{K}=\frac{1}{2}\left[R_{K} c_{K}(0)-\rho^{2} D_{R}\right],
$$

where

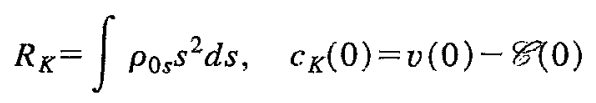

and

$$
\rho^{2} D_{K}=\int \rho_{s_{1}} \rho_{s_{2}}\left[\frac{e^{v}}{G(r)}-v-1\right] v d s_{1} d s_{2} d \mathbf{r}
$$

with $v=s_{1} s_{2} \mathscr{C}(r)$. Compared with Eq. (2.8), we here have introduced a correlation factor $G(r)$. The purpose of this factor is to quench the particle positions such that their positions are the same for all frequencies as discussed in the Introduction. $G(r)$ thus corresponds to the Boltzmann factor from a repulsive potential that will cancel induced correlations. $G(r)$ is

$$
G(r)=\frac{\int \rho_{s_{1}} \rho_{s_{2}} e^{v} d s_{1} d s_{2}}{\int \rho_{s_{1}} \rho_{s_{2}} d s_{1} d s_{2}}=\frac{a}{\left[a^{2}-\mathscr{C}^{2}(r)\right]^{1 / 2}}
$$

with Eq. (2.11) inserted for $\rho_{s}$. Likewise, $D_{K}$ in Eq. (2.15) is found to be

$$
D_{K}=D_{1 K}-D_{2 K}
$$

with

$$
D_{1 K}=\int \frac{\mathscr{C}^{2}(r)}{a^{2}-\mathscr{C}^{2}(r)} d \mathbf{r}, \quad D_{2 K}=\int \frac{\mathscr{C}^{2}(r)}{a^{2}} d \mathbf{r} .
$$

Note that $G(r)$ has the interesting effect that it removes the square root from $D_{1 K}$ by which the frequencies for a pair of particles can come out sharply for low density as we will see later.

To determine thermodynamics and frequency spectrum, we will need the full internal energy and then also $R_{K}$. Therefore we substitute $\rho_{0 s}$ in Eq. (2.14) by use of the equation of chemical equilibrium (2.6) to get $\left[v=s_{1} s_{2} \mathscr{E}(r)\right]$

$$
\begin{aligned}
R_{K}= & \int \rho_{s} s^{2} d s+\int \rho_{s_{1}} \rho_{s_{2}} s_{1}^{2}\left[\frac{e^{v}}{\dot{G}(r)}-\frac{1}{2} v^{2}-v-1\right] \\
& \times d s_{1} d s_{2} d \mathbf{r} .
\end{aligned}
$$

Use of Eq. (2.11) and use of partial integration in the last integral noting that

$$
\rho_{s_{1}} s_{1}=-(1 / a) \partial \rho_{s_{1}} / \partial s_{1}
$$

then yields

$$
R_{K}=\frac{1}{a}\left(\rho_{0}+\rho^{2} D_{K}\right)
$$

with $D_{K}$ as given by Eqs. (2.15) and (2.17). Furthermore, $\rho$ is the density of "free" particles and $\rho_{0}$ is total density [with $B$ in Eq. (2.11) suitably adjusted] given by

$$
\rho=\int \rho_{s} d s
$$




$$
\rho_{0}=\int \rho_{0 s} d s=\int \rho_{s} d s+\int \rho_{s_{1}} \rho_{s_{2}} K_{s_{1} s_{2}} d s_{1} d s_{2}
$$

with

$$
K_{s_{1} s_{2}}=\left[\frac{e^{v}}{G(r)}-\frac{1}{2} v^{2}-v-1\right] .
$$

Note that with definition (2.16) for $G(r)$, only the $v^{2}$ term will contribute in Eq. (2.20). Hence this term seems somewhat odd, and due to its effect on the frequency spectrum, we find reasons to modify it later.

Our method to find the frequency spectrum was established by Hoye and Olaussen in Ref. 6, where it was shown that the internal energy can be uniquely decomposed into that of a set of harmonic oscillators. We then will need the energy $U^{R}$ of the reference path integral quantum system. This is the system with density distribution (2.11), but with interaction (2.1) switched off. With $R_{K}$ defined by Eq. (2.14), this is found to be ${ }^{1}$

$$
\beta U^{R}=\frac{1}{2} \sum_{K}\left[\rho_{0}-\sigma\left(K^{2}-\hbar^{2} \omega^{2}\right) R_{K}\right]
$$

where $K=2 \pi n / \beta$ with $n$ integer. These values of the Fourier variable $K$ are determined by the length $\beta$ of the periodic polymers. To Eq. (2.21), we add the excess internal energy $U^{E}$ due to the interaction (2.1). This is just expression (2.13) when summed over $K$,

$$
\beta U^{E}=\beta \sum_{K} U_{K}=\frac{1}{2} \sum_{K}\left[R_{K} c_{K}(0)-\rho^{2} D_{K}\right] .
$$

Adding both contributions, the total energy per unit volume $U$ is found to be

$$
\begin{aligned}
\beta U= & \beta\left(U^{R}+U^{E}\right) \\
= & \frac{1}{2} \sum_{K}\left\{\rho_{0}+\left[\sigma\left(K^{2}+\hbar^{2} \omega^{2}\right)+c_{K}(0)\right] R_{K}\right. \\
& \left.-2 \sigma K^{2} R_{K}-\rho^{2} D_{K}\right\} \\
= & \sum_{K}\left[\frac{1}{2}\left(\rho_{0}+a R_{K}-\rho^{2} D_{K}\right)-\sigma K^{2} R_{K}\right] \\
= & \sum_{K}\left(\rho_{0}-\sigma K^{2} R_{K}\right),
\end{aligned}
$$

where Eqs. (2.11) $\left[c_{K}(0)=v(0)-\mathscr{C}(0)\right],(2.18)$, and (2.19) have been used.

One can note the simple form and consistency of the energy (2.23). The classical limit is easily recovered when $\beta \rightarrow 0$, by which the sum reduces to $K=0$ as the only term, whereby $R_{K}$ does not contribute, and the energy per particle (oscillator) becomes $U / \rho_{0}=k T$. Furthermore, Eq. (2.23) decomposes into harmonic oscillator contributions, and this means that $R_{K}$ determines the frequency spectrum. Consequently, result (2.23) is also consistent with the more common procedure of determining the energy or frequency spectrum from the Green's function.
From Eq. (2.23), the frequency spectrum $g\left(m^{2}\right)$ can be determined as follows: ${ }^{6}$

$$
f\left(K^{2}\right)=1-\frac{1}{\rho_{0}} \sigma K^{2} R_{K}=\int \frac{m^{2}}{K^{2}+m^{2}} g\left(m^{2}\right) d\left(m^{2}\right)
$$

with

$$
g\left(m^{2}\right)=-\frac{1}{\pi m^{2}} \operatorname{Im} f\left(-m^{2}+i \gamma\right) \quad(\gamma \rightarrow 0+)
$$

or

$$
g\left(m^{2}\right)=-\frac{\sigma}{\pi \rho_{0}} \operatorname{Im}\left(R_{K}\right),
$$

where one puts $K^{2}=-m^{2}+i \gamma(\gamma \rightarrow 0+)$, i.e., $K$ is imaginary. The frequency $\omega$ is given by

$$
m=\hbar \omega
$$

[compared with the reference, $g\left(\mathrm{~m}^{2}\right)$ is here redefined, i.e., $g \rightarrow m^{2} g$ ]. From Eq. (2.24), it follows that $g\left(m^{2}\right)$ is normalized in such a way that

$$
f(0)=\int g\left(m^{2}\right) d\left(m^{2}\right)=1 .
$$

\section{LOW DENSITY LIMIT}

We will now investigate the properties of the spectrum in the low density limit, with the second virial coefficient included, to see how the exact answer comes out. From Eq. (2.2), we then have $\left[\tilde{\mu}(k)=\rho_{0}+\cdots\right]$ by inserting the forms (2.12) and using Eqs. (2.14), (2.18), and (2.19)

$$
\begin{aligned}
& \tilde{\mathscr{C}}(k)=\tilde{v}(k)+\tilde{v}(k) R_{K} \tilde{v}(k)+\cdots, \\
& c_{K}(0)=v(0)-\mathscr{E}(0)=-\frac{\rho_{0}}{a} \int v^{2}(r) d \mathbf{r} .
\end{aligned}
$$

From Eq. (2.17), we find $[\mathscr{E}(r)=v(r)+\cdots]$

$D_{1 K}=\int \frac{v^{2}}{a^{2}-v^{2}} d \mathbf{r}, \quad D_{2 K}=\frac{1}{a^{2}} \int v^{2} d \mathbf{r}=-\frac{1}{\rho_{0} a} c_{K}(0)$,

where $v=v(r)$. Thus Eq. (2.19) becomes, using Eqs. (2.11) and $(2.14)\left(\rho_{0} \rightarrow \rho\right)$

$$
R_{K}=\frac{\rho\left[1+c_{K}(0) / a\right]}{a_{0}+c_{K}(0)}+\frac{\rho^{2}}{a} D_{1 K}
$$

with $a_{0}=\sigma\left(K^{2}+\hbar^{2} \omega_{0}^{2}\right)$. Since $a \rightarrow a_{0}$ as $\rho \rightarrow 0, c_{K}(0)$ almost cancels out in the first term. For computation of thermodynamic quantities, the difference between $a_{0}$ and $a$ in Eq. (3.3) is not significant since $c_{K}(0)$ or $D_{2 K}$ are perturbing terms. However, for the frequency spectrum, this difference is more important and details of the spectra are sensitive to small changes in these quantities. $a_{0}$ alone will yield the sharp frequency $\omega_{0}$. When doing the MSA, $c_{k}(0)$ in the denominator will broaden the sharp frequency into a rounded off peak of width $\approx \sqrt{\rho}$. Also for imaginary $K$ that determines frequencies, $c_{K}(0)$ will grow into the magnitude of $a_{0}$ by which the difference $a-a_{0}$ will no longer be negligible. This has the effect that by including $c_{k}(0)$ in the 
numerator, the frequency spectrum will get a negative part at the ends of the rounded peak. Negative values are of course unphysical, but correctly make the effective width close to zero, since for $\rho \rightarrow 0$, the proper width will be taken care of by the frequencies of the $D_{1 K}$ term which we will identify below. Then to sharpen up the peak more properly, the $c_{K}(0)$ term in the numerator can be expanded to first order, by which the two $c_{K}(0)$ terms cancel. By that,

$$
R_{K}=\frac{\rho}{a_{0}}+\frac{\rho^{2}}{a_{0}} D_{1 K},
$$

using $a \sim a_{0}$ in the perturbing last term. Inserting expression (3.2) for $D_{1 K}$, one can expand in partial fractions to obtain

$$
R_{K}=\frac{\rho}{a_{0}}+\rho^{2} \int\left[\frac{1}{2}\left(\frac{1}{a_{0}-v}+\frac{1}{a_{0}+v}\right)-\frac{1}{a_{0}}\right] d \mathbf{r} .
$$

This result is easily interpreted in relation to the single oscillator frequency from the first term, while the $a_{0} \pm v$ terms in the integrand are the frequencies from coupled pairs of oscillators at varying separation. These oscillator pairs of course reduce the density of single oscillators, and this is taken care of by the last term. With $a_{0}$ given below Eq. (3.3), these pairs of oscillators will have frequencies

$$
\omega^{2}=\omega_{0}^{2} \pm \frac{1}{M} v(r) \quad\left(\sigma \hbar^{2}=M\right) .
$$

Thus we have recovered the exact result at low densities. Note that this is crucially dependent on the introduction of the "quenching" factor $G(r)$ in Eq. (2.15) when treating each frequency separately. Since the function $G(r)$ is not a constant, it may bring out some inconsistency, but this possibility will be disregarded. As mentioned in the Introduction, the low density result was already derived by Gibbons et al. ${ }^{14}$ and Bush et al. ${ }^{15}$

\section{CORRECTIONS FOR ARBITRARY DENSITY}

The equations above were derived for low density with respect to the perturbing term $D_{K} \cdot f_{i j}(r)$ in Eq. (2.5) is a two body Boltzmann type term with simple $\rho$ vertices (disregarding that $\rho_{0 i}$ is replaced with $\rho_{i}$ ). In view of $\gamma$ ordering, this can only be valid at low density. At higher density, it is necessary to introduce hypervertices that depend on the properties of the reference system (unless the latter is an ideal gas). For one-component systems, the use of hypervertices is well known. ${ }^{17,21}$ This can be generalized to multicomponent systems. ${ }^{22}$ Certain hypervertices can be directly related to the equation of state for the reference system, at least as long as they can be regarded as $\delta$ functions compared with the range of the perturbing interaction. These graphs represent $n$-particle cluster functions whose integrals follow from the equation of state (compressibility relations). Due to symmetry, vertices with an odd number of root points will vanish in the present case, as vertices are integrated along with a factor $s$ from each chain bond attached to the vertex. In the present instance, the reference system has a density distribution $\rho_{0 s} \approx \rho_{s}$ that is Gaussian and given by Eq. (2.11). (Note that in density expansions, the densities $\rho_{i} \leftrightarrow \rho_{s}$ are those of the perturbed system.) With interaction (2.1) switched off, all particles are in fact identical, and the equation of state will be that of the one-component system. The integrated vertices for the multicomponent system can now be expressed in terms of the one-component equation of state. With $\rho_{s}$ given by Eq. (2.11), one then has for a $2 n$ point vertex ${ }^{22}$

$$
A_{2 n}=\frac{(2 n) !}{2^{n} n ! a^{n}} B_{2 n}, \quad B_{2 n}=\frac{\partial^{n}(\beta p)}{\partial(\beta g)^{n}},
$$

where $p$ and $g$ are the pressure and chemical potential of the one component system at density $\rho$. The prefactor of Eq. (4.1) is the Gaussian average of $s^{2 n}$, and in the low density limit, the second factor is the ideal gas result $p$ in accordance with our previous results.

For $n=1$, we have the simple $\rho$ vertex as $\partial(\beta p) / \partial(\beta g)$ $=\rho$, and for $n=2$, we get $(\beta=$ constant $)$

$$
B_{4}=\mu=\frac{\partial \rho}{\partial(\beta g)}=\left[\frac{\partial(\beta g)}{\partial \rho}\right]^{-1}=\rho\left[\frac{\partial(\beta p)}{\partial \rho}\right]^{-1}
$$

With $\mu=\mu(\rho)$, one then obtains further

$$
B_{6}=\frac{\partial \mu}{\partial \beta g}=\mu^{\prime} \mu, \quad \mu^{\prime}=\frac{\partial \mu}{\partial \rho},
$$

etc., or generally

$$
B_{2(n+1)}=B_{2 n}^{\prime} \mu \text {. }
$$

To simplify, we will use $B_{4}$ for all $B_{2 n}$ with $n \geqslant 2$ as this term will be the major contribution by $\gamma$ ordering, and anyway, all $B_{2 n}$ 's become equal in the low density limit and they will all vanish at close packing. With this simplification, we now can go through Eqs. (2.5)-(2.7) and (2.15)-(2.20). The only resulting change will be that $\rho^{2}$ is then replaced by $\mu^{2}$ in the perturbing terms. The original derivations of Eqs. (2.3)(2.9) will be unchanged as long as $\mu$ depends only on density. This is the case for systems with strict hard cores like hard spheres. The result (4.2) for $B_{4}$. [and more generally Eqs. (4.3)] can be understood or seen by more direct consideration. For a one component fluid, $B_{2 n}$ above will represent irreducible $n$-point vertices. With an interaction of type (2.1), whose average with density distribution (2.11) is zero, an even number of potential bonds must meet at each root point to give a net contribution. Thus, the integrated $2 n$-point function becomes effectively an $n$-point function (i.e., the number of root points will vary between 1 and $n$ ). With one root point (the simple $\rho$ vertex), all potential $2 n$ bonds meet at the same point. This will give the low density value $B_{2 n}=\rho$, and the prefactor of $B_{2 n}$ in Eq. (3.4) is the Gaussian average $\left\langle s^{2 n}\right\rangle$. For $B_{4}$, there also will be graphs with two root points that clearly represent the pair correlation function of the one-component reference system. At each root point, the factor $\left\langle s^{2}\right\rangle$ is produced. The four potential (or chain) bonds can pair up in three different combinations. Altogether the prefactor $3\left\langle s^{2}\right\rangle^{2}$ is produced, and with Gaussian density distribution, this equals $\left\langle s^{4}\right\rangle$. So adding the two contributions, result (4.1) together with Eq. (4.2) must come out.

Vertices with more that one root point make result (2.19) from the partial integration of Eq. (2.18) questionable, as the latter will not follow from the former alone. However, a compensation will enter the density distribution that follows 
from Eq. (2.9). Introducing more general density dependent vertices instead of the simple $\rho_{i}$ vertex in Eqs. (2.5)-(2.7), one will find a remaining $K_{i j}$ term in the chemical potential (2.9) following the derivations of Ref. 18. Hence, we use Eq. (2.19) replacing $\rho^{2}$ with $\mu^{2}$. Also as explained in connection with Eqs. (3.3) and (3.4), the $D_{2 K}$ term is further expanded into the denominator. Then Eq. (2.19) becomes

$$
R_{K}=\frac{1}{a}\left(\rho_{0}-\mu^{2} D_{2 K}+\mu^{2} D_{1 K}\right) \approx \frac{1}{a_{e}}\left(\rho_{0}+\mu^{2} D_{1 K}\right)
$$

with

$$
a_{e}=a\left(1+\frac{1}{\rho_{0}} \mu^{2} D_{2 K}\right),
$$

and from Eq. (2.11)

$$
\begin{gathered}
a=a_{0}+c_{K}(0), \quad a_{0}=\sigma\left(K^{2}+\hbar^{2} \omega_{0}^{2}\right), \\
c_{K}(0)=-(\mathscr{C}(0)-v(0)) .
\end{gathered}
$$

$a_{e}$ can now replace $a$ in Eq. (2.11). Consistent with this, $D_{2 K}$ is taken out of the equations of chemical equilibrium. This means removing the $v^{2}$ terms of Eqs. (2.15) and (2.20). By that, the latter integral of Eq. (2.20) also will be zero, such that

$$
\rho_{0}=\rho \text {. }
$$

In accordance with this, $a$ of Eqs. (2.16)-(2.17) is also replaced by $a_{e}$.

To summarize our equations to determine frequency spectra or thermodynamics (internal energy), we have now, first of all, Eq. (4.4) with Eq. (4.5) inserted, so we can write

$$
R_{K}=\frac{1}{a_{e}}\left(\rho+\mu^{2} D_{1 K}\right)
$$

with $a_{e}$ given by Eq. (4.4) and $D_{2 K}$ and $D_{1 K}$ given by Eq. (2.17), or Eq. (6.1) for the continuum case. As mentioned above, the two-point vertex function $\mu$ is obtained from the equation of state of the reference system via Eq. (4.2) as long as its extension can be regarded as a $\delta$ function. Otherwise, one should replace it with the appropriate pair-correlation function (added to the simple $\rho$ vertex) connecting its root points by four $\mathscr{E}$ bonds in the ways possible. This will give a more complicated numerical problem and we will not go into it here. With $R_{K}$ at hand, the internal energy is then given by Eq. (2.23), and if the chain bond $\mathscr{C}(r)$ is replaced by its MSA value, then no more equations are needed and numerical solution should be straightforward.

However, if perturbed values of $\mathscr{E}(r)$ in accordance with Eqs. (2.2) - (2.6) are considered, then the numerical problem becomes more involved, especially if the core condition $h(r)=0$ (for the $s_{1} s_{2}$ component of the pair correlation function) is imposed by adjusting $v(r)$ inside the core. The solution of the latter problem is not yet obvious. For example, Høye and Lomba encountered numerical instabilities beyond a certain density and below a certain temperature in a related problem for electrolytes. ${ }^{23}$ Anyway, we may write down the appropriate equations for the correlation function: Equation (2.5) becomes

$$
\mu_{s_{1} s_{2}}(r)=\rho_{0 s_{1}} \delta_{s_{1} s_{2}}+\rho_{s_{1}} \rho_{s_{2}}\left[\frac{e^{v}}{G(r)-v}-1\right]
$$

$\left[v=s_{1} s_{2} \mathscr{E}(r)\right]$. The factor $G(r)$ is introduced as done in Eq. (2.15).] At the vertices, Eq. (4.7) is multiplied with $s_{1} s_{2}$ and integrated to give

$$
\tilde{\mu}(k)=R_{K}+\rho^{2} \int\left[\frac{\mathscr{E}(r)}{a_{e}^{2}-\mathscr{C}_{(r)}^{2}}-\frac{\mathscr{E}(r)}{a_{e}^{2}}\right] e^{i \mathbf{k r}} d \mathbf{r}
$$

using Eqs. (2.14)-(2.17). To determine $\mathscr{E}(r)$ or $h(r)$, this is to be used along with Eqs. (2.2) and (2.4) with $v(r)$ and $\mathscr{E}(r)$ as defined by Eq. (2.12). (That is, the form of these equations is unchanged except that now $\tilde{v}(k)$ and $\tilde{c}(k)$ are no longer matrices.)

\section{COMPARISON WITH AN EXACTLY SOLVABLE CASE}

Hübner, Høye, and Olaussen studied an exactly solvable model in one dimension, ${ }^{24}$ and the MSA solution was compared with the exact one. The model consisted of harmonic oscillators randomly located on a one dimensional lattice with nearest neighbor interactions. When referring to the equations of Ref. 24, we will precede them by the numeral I.

For cells of unit length, one from Eqs. (I3.9), (I4.3), or (I4.5) finds for the nearest neighbor interaction

$$
v(r) \rightarrow v( \pm 1)=\frac{\epsilon}{2 \alpha},
$$

where $1 / \alpha=M \omega_{0}^{2}\left(M=m_{r}\right.$ of the reference). $\epsilon$ measures the relative strength of interaction and is limited to $|\epsilon| \leqslant 1$ to have stability. By use of Eq. (3.5), the exact result will be recovered in the low density limit as is easily verified. First Eq. (3.6) yields the pair of frequencies

$$
\omega^{2}=\omega_{0}^{2} \pm \frac{1}{M} v( \pm 1)=\omega_{0}^{2}\left(1 \pm \frac{1}{2} \epsilon\right)
$$

which are precisely the two eigenfrequencies of a pair of particles [e.g., see Eq. (13.14)]. For the integral in Eq. (3.5), we now obtain a sum and we get

$R_{K}=\rho(1-2 \dot{\rho}) \frac{1}{a_{0}}+\rho^{2}\left[\frac{1}{a_{0}-v( \pm 1)}+\frac{1}{a_{0}+v( \pm 1)}\right]$.

Here the first term represents single oscillators, while the latter two represent pairs of oscillator $s$. Their densities coincide with the exact ones to second power in $\rho$, as from Eq. (13.17), one has $\rho(1-\rho)^{2}=\rho(1-2 \rho+\cdots)$ and $\rho^{2}(1-\rho)^{2}=\rho^{2}+\cdots$. To the extent $R_{K}$ is exact, the internal energy (2.23) will be exact too.

From graph analysis, we expect our result to be exact to the power $\epsilon^{4}$ since graphs with up to four potential bonds are included in full for arbitrary density, i.e., this should hold for thermodynamic quantities such as the internal energy that can be expanded in powers of $\epsilon^{2}$. However, the frequency spectrum cannot be expanded in this way, especially when the exact one is discrete. In the Appendix, we perform an expansion of the internal energy to the power $\epsilon^{4}$ and we find 
full agreement with the exact result for $T=0$ comparing Eqs. (A13) and (A20). This comparison can also be extended to arbitrary $T$ by considering $R_{K}$. A comparison of Eq. (A14) with Eq. (A23) then shows agreement to the power $\epsilon^{4}$ when Eq. (A16) is inserted.

To compare for arbitrary $\epsilon$, some numerical work will be required. $\epsilon$ is limited to $|\epsilon| \leqslant 1$ due to stability (i.e., for $|\epsilon|=1$ the lowest eigenfrequency is $\omega=0$ ). For the extreme case of $\epsilon=1$, the exact and MSA internal energies per particle are then compared in Figs. 1 and 2 of Ref. 24. Their small difference is plotted in Fig. 2 of Ref. 24. For small densities, the limiting form (5.3) yields the exact result as explained before. The correction to the MSA solution is then close to Eq. (A20) \{i.e., replacing $5 \epsilon^{4} / 1024$ with $\sqrt{1-\epsilon / 2}+\sqrt{1+\epsilon / 2}$ $-\epsilon^{2} / 16=5 \epsilon^{4} / 1024+\cdots$ makes Eq. (A20) exact for small $\rho$ [see Eq. (I5.10)]\}. For larger $\rho$, Eq. (A20) will deviate further from the exact result, mainly because $\mathscr{E}(r)$ will differ more from $v(r)$. Now we want to take this into account utilizing the MSA value for the chain bond $\mathscr{R}(r)$, which then also is the MSA pair correlation function $h(r)$, i.e., Eq. (2.4) with simple $\rho$ vertices. With form (2.12), the $\rho$ vertices give factors $R_{K}$. The core condition and nearest neighbor interaction implies that

$$
\Gamma(n)=R_{K} \sigma^{|n|},
$$

where $\tilde{\Gamma}(k)=R_{K}+R_{K}^{2} \tilde{\mathscr{E}}(k)$ [E(0)=0]. [Note $\sigma$ in Eq. (5.4) is a different quantity than the one defined by Eq. (2.10).] This form is verified by Fourier transform of Eq. (5.4), which yields the integrand of Eq. (I4.8) [ $\left.\tilde{\mathscr{C}}=\tilde{h}^{K}(k)\right]$. This also determines $\sigma$ and its relation to the MSA $R_{K}$,

$$
\sigma=-\frac{\alpha}{\epsilon} c_{K}(0), \quad R_{K}=\frac{\rho}{a}=\frac{\alpha}{\epsilon} \frac{2 \sigma}{1-\sigma^{2}} .
$$

[The latter is in accordance with Eq. (14.11) and $c_{K}(0)=c$ of Eq. (I4.7).] $c_{K}(0)$ is given by Eq. (A22). Equation (5.4) means that

$$
\mathscr{E}(n)=\frac{1}{R_{K}} \sigma^{|n|} \quad(n \neq 0)
$$

or

$$
\mathscr{Z}(n) / a=\frac{1}{\rho} \sigma^{|n|},
$$

which inserted in Eqs. (2.17) yields

$$
D_{1 K}=2 \sum_{n=1}^{\infty} \frac{\left(\sigma^{n} / \rho\right)^{2}}{1-\left(\sigma^{n} / \rho\right)^{2}}, \quad D_{2 K}=2 \frac{(\sigma / \rho)^{2}}{1-\sigma^{2}}
$$

Equations (5.5) and (5.8) inserted in (4.4) give $\left(\rho_{0}=\rho\right)$

$$
\begin{aligned}
a_{e} & =a\left[1+\rho(1-\rho)^{2} D_{2 K}\right] \\
& =a_{0}+c_{K}(0)+\rho(1-\rho)^{2} a D_{2 K} \\
& =a_{0}-\frac{\epsilon}{\alpha} \rho(2-\rho) \sigma
\end{aligned}
$$

with

$$
a=a_{0}-\frac{\epsilon}{\alpha} \sigma .
$$

The deviation of $R_{K}$ from its MSA value is then $[\mu=\rho(1-\rho)]$

$$
\Delta R_{K}=R_{K}-\frac{\rho}{a}=\rho\left[\frac{1}{a_{e}}\left(\rho+\mu^{2} D_{1 K}\right)-\frac{1}{a}\right]
$$

with expressions (A22), (4.4), (5.8), and (5.9) inserted. This again determines the deviation of the internal energy from its MSA value. Numerical integration such as Eq. (A17) with Eq. (5.10) inserted yields this deviation per particle $\Delta u$ at $T=0$. Comparing with Fig. 2 of Ref. 24, the exact slope of the curve is recovered as $\rho \rightarrow 0$, in accordance with Eq. (5.3). The extreme case $\epsilon=1$ is considered, and even then, $\Delta u$ follows the exact result closely and cannot be distinguished from it on the figure up to density $\rho \approx 0.4$. For higher densities, its magnitude is slightly less than the exact value. This difference grows to about $10^{-4} u_{0}$ in the region $\rho \approx 0.7-0.9$, while the maximum value of $\Delta u$ is about $17 \times 10^{-4} u_{0}$ near $\rho \approx 0.5\left(u_{0}=\hbar \omega_{0} / 2\right)$. Thus we can conclude that our correction $\Delta u$ to the MSA result improves it considerably, leading to results close to the exact one.

We will also study some features of the frequency spectrum that according to Eq. (2.25) is determined by the imaginary part of $R_{K}$. Let us consider then the first term of Eq. (4.6). With $a$ in place of $a_{e}$, this would yield the MSA spectrum (A9) or (I4.23) with $q$ given by (A1). Use of $a_{e}$ given by Eq. (5.9) will change this result into one similar to Eq. (A9), except that the denominator $\rho\left(1-q^{2}\right)$ is replaced by $\rho^{2}(2-\rho)+(1 / \rho-2) q^{2}$. Thus, as discussed in connection with Eqs. (3.5) and (5.3), the frequency distribution will be sharply peaked at $q=0$ for low density. (Its height will be $\sim \rho^{-3 / 2}$ and its width will be $\sim \rho^{3 / 2}$.) But as in the MSA, the frequencies are still spread out through the region $q^{2}<\rho(2-\rho)$. The last term of Eq. (4.6) with Eq. (5.8) inserted represents a pair of oscillators for each value of $n$ in the sum like the last term of Eq. (5.3). However, for $n \geqslant 2$, the $\sigma^{n} / \rho=1$ condition cannot be fulfilled for $q$ real. Thus contributions for $n \geqslant 2$ will be smoothed out inside the MSA region $q^{2}<\rho(2-\rho)$, where $\sigma$ is complex and consequently we have an imaginary part. For $n=1$, one finds using Eqs. (5.5) and (A22) that $\left(a_{0}=\epsilon q / \alpha\right) \sigma / \rho=1$ for $q=(1+2 \rho$ $\left.-\rho^{2}\right) / 2>\sqrt{\rho(2-\rho)}$, and a pair of sharp frequencies outside the MSA region is obtained. In the continuum case, this latter sharp frequency will be smoothed out too due to the integral (2.17) for $D_{1 K}$, i.e., frequencies change continuously with $r$.

\section{CONTINUUM CASE}

As understood from the previous derivations, our equations also hold for the continuum case. However, we left out a leading contribution beyond the MSA due to spatial correlations between pairs of particles. This, however, can be easily taken care of by incorporating them into Eqs. (2.16) and (2.17) for $D_{1 K}$ and $D_{2 K}$. The hypervertex approximation studied by Hoye and Stell contains this contribution. ${ }^{25} \mathrm{Al}-$ ready in Eq. (2.15) a dividing factor $G(r)$ was introduced to cancel "induced" correlations. So, clearly to account for correlations, the pair distribution function $g_{0}(r)=1+h_{0}(r)$ for the reference system should multiply the $e^{v}$ factor of Eq. (2.15). Accordingly, this factor will then also enter the inte- 
grand for $D_{1 K}$ in Eq. (2.17). For the $\mathscr{G}^{2}(r)$ term of $D_{1 K}$, this contribution will be exact for all $\rho$. However, with Eq. (4.6), $\rho$ is modified with the $\mu$ factor. In order to preserve Eq. (4.6) and still keep the $8^{2}(r)$ term exact, part of it can be transferred to $D_{2 K}$. With this, we have

$$
\begin{aligned}
& D_{1 K}=\int \frac{\mathscr{E}^{2}(r)}{a^{2}-\mathscr{E}^{2}(r)} g_{0}(r) d \mathbf{r}, \\
& \mu^{2} D_{2 K}=\int \frac{\mathscr{E}^{2}(r)}{a^{2}}\left[\mu^{2} g_{0}^{\prime}(r)-\rho^{2} h_{0}(r)\right] d \mathbf{r} .
\end{aligned}
$$

With this change, Eq. (4.6) can be used as before to compute internal energy and frequency spectra. A similar type of correction to the MSA was also introduced by Winn and Kahl in their calculation of optical absorption bands in fluids. ${ }^{26} \mathrm{How}-$ ever, the $\mu$ factor that dampens the effect of pairs for higher densities has, to our knowledge, not been present in earlier treatments.

\section{ACKNOWLEDGMENTS}

The authors would like to thank Dr. M. Winn and Dr. G. Kahl for making available to them Ref. 26 prior to publication. E. L. acknowledges support from the Spanish Dirección General de Investigación Científica y Técnica (DGICYT) under Grant No. PB91-0110.

\section{APPENDIX}

We want to compare the exact internal energy and our approximate internal energy for the exactly solvable onedimensional model at $T=0$. With Eq. (I4.21), we replace our $\omega$ with

$$
q=\frac{1}{\epsilon}\left(1-\frac{\omega^{2}}{\omega_{0}^{2}}\right)
$$

and the internal energy per oscillator becomes

$$
\begin{aligned}
u & =\frac{1}{2} \hbar\langle\omega\rangle \\
& =u_{0}\langle\sqrt{1-\epsilon q}\rangle \\
& =u_{0}\left\langle\left(1-\frac{1}{8} \epsilon^{2}\left\langle q^{2}\right\rangle-\frac{5}{128} \epsilon^{4}\left\langle q^{4}\right\rangle-\cdots\right)\right\rangle,
\end{aligned}
$$

where the odd powers vanish due to symmetry. The brackets denote averages and $u_{0}=\hbar \omega_{0} / 2$. For a segment consisting of $n$ units, the exact frequencies are given by Eq. (I5.1)

$$
q=\cos \left(\frac{k}{n+1} \pi\right), \quad k=1,2, \ldots, n .
$$

The number $P_{n}$ of segments of $n$ units is given by Eq. (I3.17) ( $N$ is the number of available positions)

$$
P_{n}=N \rho(1-\rho)^{2} \rho^{n-1} \text {. }
$$

From these equations, one obtains Eq. (I5.4)

$$
\left\langle q^{2}\right\rangle=\frac{1}{2} \rho \text {. }
$$

$$
\sum_{k=1}^{n} \exp \left(i m \frac{k}{n+1} \pi\right)=-1
$$

for $m$ even and $0<m<2(n+1)$. Thus for $n \geqslant 1$,

$$
\begin{aligned}
c_{n} & =\sum_{k=1}^{n} \cos ^{4}\left(\frac{k}{n+1} \pi\right) \\
& =\sum_{k=1}^{n} \frac{1}{16}\left[\exp \left(i \frac{k}{n+1} \pi\right)+\exp \left(-i \frac{k}{n+1} \pi\right)\right]^{4} \\
& =\frac{1}{16}(-1-4+6 n-4-1)=\frac{1}{8}(3 n-5) .
\end{aligned}
$$

Altogether by use of Eqs. (A4) and (A6), we obtain ( $\left.c_{1}=0\right)$

$$
\left\langle q^{4}\right\rangle_{\mathrm{ex}}=(1-\rho)^{2} \sum_{n=2}^{\infty} \rho^{n-1} c_{n}=\frac{1}{8} \rho(1+2 \rho) .
$$

Inserted in Eq. (A2), this yields for the exact result

$$
u_{\mathrm{ex}}=u=u_{0}\left[1-\frac{1}{16} \epsilon^{2} \rho-\frac{5}{1024} \epsilon^{4} \rho(1+2 \rho)+\cdots\right] .
$$

The corresponding MSA result is obtained by use of the frequency distribution (I4.23)

$$
\begin{aligned}
& \hat{g}(q)=\frac{1}{\pi \rho} \frac{\sqrt{\rho(2-\rho)-q^{2}}}{1-q^{2}}, \quad q^{2}<\rho(2-\rho), \\
& \int \hat{g}(q) d q=1 .
\end{aligned}
$$

Use of Eq. (A9) will also give the result (A5). To obtain $\left\langle q^{4}\right\rangle$, this can be utilized

$$
\begin{aligned}
\left\langle q^{4}\right\rangle_{\mathrm{MSA}} & =\int q^{4} \hat{g}(q) d q \\
& =\int\left[q^{2}\left(q^{2}-1\right)+q^{2}\right] \hat{g}(q) d q \\
& =-\frac{1}{\pi \rho} \int q^{2}\left[\rho(2-\rho)-q^{2}\right]^{1 / 2} d q+\frac{1}{2} \rho .
\end{aligned}
$$

The last integral is integrated by substitution of $q$ $=\sqrt{\rho(2-\rho)} \sin \phi$, and we find

$$
\left\langle q^{4}\right\rangle_{\mathrm{MSA}}=-\frac{1}{8} \rho(2-\rho)^{2}+\frac{1}{2} \rho=\frac{1}{8} \rho^{2}(4-\rho),
$$

which yields the MSA result

$$
U_{\mathrm{MSA}}=u_{0}\left[1-\frac{1}{16} \epsilon^{2} \rho-\frac{5}{1024} \epsilon^{4} \rho^{2}(4-\rho)+\cdots\right] .
$$

For the difference between Eqs. (A8) and (A12), we obtain

$$
U_{\mathrm{ex}}-U_{\mathrm{MSA}}=-u_{0} \frac{5}{1024} \epsilon^{4} \rho(1-\rho)^{2}+\cdots .
$$

To obtain $\left\langle q^{4}\right\rangle_{\text {ex }}$, we first note that 
To find our correction to the MSA result, one goes back to Eq. (4.6) or Eq. (2.19). For small $\epsilon$, this correction reduces to the last term of Eq. (2.19), and by further use of Eqs. (2.17) and (5.1), this reduces to

$$
\Delta R_{K}=\frac{1}{a} \mu^{2} D_{K} \rightarrow \frac{\mu^{2}}{a_{0}} \sum_{r}\left[\frac{v(r)}{a_{0}}\right]^{4}=2 \frac{\mu^{2}}{a_{0}}\left(\frac{\epsilon}{2 \alpha a_{0}}\right)^{4}
$$

as $\mathscr{C}(r) \rightarrow v(r)$ and $a \rightarrow a_{0}$ in this limit. In accordance with Eq. (4.6), the $\rho^{2}$ factor of Eq. (2.19) has been replaced by the factor $\mu^{2}$. The latter is obtained via the equation of state for the ideal lattice gas that forbids multiple occupancy (hard cores), but otherwise there is no interaction. Its pressure $p$ is given by (with cells of unit volume)

$$
\beta p=-\log (1-\rho)
$$

from which Eq. (4.2) gives

$$
\mu=\rho\left[\frac{\partial(\beta p)}{\partial \rho}\right]^{-1}=\rho(1-\rho) .
$$

Together with Eq. (2.23), one then finds for the correction to the internal energy per particle $(T=0)$

$\Delta u=-\frac{1}{\beta \rho} \sum_{K} \sigma K^{2} \Delta R_{K} \underset{\beta \rightarrow \infty}{\longrightarrow}-\frac{1}{8} \rho(1-\rho)^{2} \epsilon^{4} \hbar \omega_{0}\left(\mathrm{I}_{4}-\mathrm{I}_{5}\right)$

with

$$
I_{n}=\frac{1}{2 \pi} \int_{-\infty}^{\infty} \frac{d x}{\left(x^{2}+1\right)^{n}},
$$

where $x=K /\left(\hbar \omega_{0}\right)$ has been introduced. $a_{0}$ is given by Eq. (4.4). ( $K=2 \pi n / \beta$ with the $n$ integer.) With

$$
I_{1 \xi}=\frac{1}{2 \pi} \int_{-\infty}^{\infty} \frac{d x}{x^{2}+\xi}=\frac{1}{2 \sqrt{\xi}},
$$

one easily finds

$$
I_{n \xi}=\frac{1}{(n-1) !}\left(-\frac{\partial}{\partial \xi}\right)^{n-1} I_{1 \xi}
$$

or $(\xi=1)$,

$$
\begin{aligned}
& I_{n+1}=\frac{2 n+1}{2 n} I_{n}=\frac{1}{2} \frac{(2 n) !}{\left(2^{n} n !\right)^{2}}, \\
& I_{4}=\frac{1}{2} \cdot \frac{1}{2} \cdot \frac{3}{4} \cdot \frac{5}{6}=\frac{5}{32}, \\
& I_{4}-I_{5}=\left(1-\frac{7}{8}\right) I_{4}=\frac{5}{256},
\end{aligned}
$$

which inserted in Eq. (A17) yields $\left(u_{0}=\hbar \omega_{0} / 2\right)$

$$
\Delta u=-\frac{5}{1024} \rho(1-\rho)^{2} \epsilon^{4} u_{0}
$$

This is precisely the difference (A13) to order $\epsilon^{4}$. Our correction is thus exact to this order.

We can also check this correction for arbitrary temperature by expanding $R_{K}$ in powers of $\epsilon$. For the exact solution, one will obtain by expansion

$\frac{1}{\rho} R_{K}^{\mathrm{ex}}=\left\langle\frac{1}{a_{\mathrm{ex}}}\right\rangle=\frac{1}{a_{0}}\left[1+\left(\frac{\epsilon}{a_{0} \alpha}\right)^{2}\left\langle q^{2}\right\rangle+\left(\frac{\epsilon}{a_{0} \alpha}\right)^{4}\left\langle q^{4}\right\rangle_{\mathrm{ex}}+\cdots\right]$

Here

$$
\begin{aligned}
a_{\mathrm{ex}} & =\sigma\left(K^{2}+\hbar^{2} \omega^{2}\right) \\
& =\sigma\left[K^{2}+\hbar^{2} \omega_{0}^{2}(1-\epsilon q)\right] \\
& =a_{0}\left(1-\frac{1}{a_{0} \alpha} \epsilon q\right) \quad\left(\sigma \hbar^{2} \omega_{0}^{2}=1 / \alpha\right) .
\end{aligned}
$$

For the MSA solution, one obtains from Eqs. (14.6) and (I4.13)

$$
\begin{aligned}
& c_{K}(0)=\frac{1}{2-\rho}\left[-a_{0}+\sqrt{a_{0}^{2}-\rho(2-\rho)\left(\frac{\epsilon}{\alpha}\right)^{2}}\right]=\frac{-\rho(\epsilon / \alpha)^{2}}{a_{0}+\sqrt{a_{0}^{2}-\rho(2-\rho)(\epsilon / \alpha)^{2}}}=-\frac{1}{2}\left(\frac{\epsilon}{a_{0} \alpha}\right)^{2}\left[1+\frac{1}{4} \rho(2-\rho)\left(\frac{\epsilon}{a_{0} \alpha}\right)^{2}+\cdots\right] \\
& \frac{1}{\rho} R_{K}^{\mathrm{MSA}}=\frac{1}{a_{0}+c_{K}(0)}=\frac{1}{a_{0}}\left[1+\frac{1}{2} \rho\left(\frac{\epsilon}{a_{0} \alpha}\right)^{2}+\frac{1}{8} \rho^{2}(4-\rho)\left(\frac{\epsilon}{a_{0} \alpha}\right)^{4}+\cdots\right] .
\end{aligned}
$$

The difference becomes

$$
\Delta R_{K}=R_{K}^{\mathrm{ex}}-R_{K}^{\mathrm{MSA}}=\frac{1}{8 a_{0}} \rho^{2}(1-\rho)^{2}\left(\frac{\epsilon}{a_{0} \alpha}\right)^{4}
$$

This is precisely correction (A14). 
'J. S. Hbye and G. Stell, J. Chem. Phys. 75, 5133 (1981).

${ }^{2}$ M. J. Thompson, K. S. Schweitzer, and D. Chandler, J. Chem. Phys. 76, 1128 (1982).

${ }^{3}$ L. R. Pratt, Mol. Phys. 40, 347 (1980).

${ }^{4}$ J. S. Hoye and G. Stell, J. Chem. Phys. 73, 461 (1980).

${ }^{5}$ R. P. Feynmann and A. R. Hibbs, Quantum Mechanics and Path Integrals (McGraw-Hill, New York, 1965).

${ }^{6}$ J. S. Høye and K. Olaussen, J. Chem. Phys. 77, 2583 (1982).

${ }^{7}$ D. Chandler, K. Schweitzer, and P. G. Wolynes, Phys. Rev. Lett. 49, 1100 (1982).

${ }^{8}$ R. Stratt, Annu. Rev. Phys. Chem. 41, 175 (1990), and references therein.

${ }^{9}$ D. E. Logan and M. D. Winn, J. Phys. C 21, 5773 (1988); M. D. Winn and D. E. Logan, J. Phys. Condensed Matter 1, 8683 (1989); see also C. F. Strnadl and G. Kahl, ibid. 5, 6801 (1993).

${ }^{10} \mathrm{Z}$. Chen and R. Stratt, J. Chem. Phys. 97, 5687 (1992); 97, 5692 (1992).

${ }^{11}$ J. S. Hoye and G. Stell, J. Chem. Phys. 77, 5173 (1982).

${ }^{12}$ J. S. Hoye and E. Lomba, J. Chem. Phys. 93, 4272 (1990).

${ }^{13} \mathrm{~K}$. Ganguly and R. Stratt, J. Chem. Phys. 95, 4418 (1991).
${ }^{14}$ M. K. Gibbons, D. E. Logan, and O. A. Madden, Phys. Rev. B 38, 7292 (1988).

${ }^{15}$ I. J. Bush, D. E. Logan, P. A. Madden, and M. D. Winn, J. Phys. Condensed Matter 1, 2551 (1989).

${ }^{16}$ Y. Wan and R. M. Stratt. J. Chem. Phys. 100, 5123 (1994).

${ }^{17}$ P. C. Hemmer, J. Math. Phys. 5, 75 (1964); J. L. Lebowitz, G. Stell, and S. Baer, ibid. 6, 1282 (1965).

${ }^{18}$ J. S. Høye and K. Olaussen, Phys. Status Solidi A 104, 447 (1980).

${ }^{19}$ J. M. Kosterlitz and D. J. Thouless, J. Phys. C 6, 1181 (1973); J. M. Kosterlitz, ibid. 7, 1046 (1974); J. S. Hǿye and K. Olaussen, ibid. 107, 241 (1981).

${ }^{20}$ L. Onsager, Ann. N.Y. Acad. Sci. 51, 627 (1949).

${ }^{21}$ J. S. Hbye, Ph.D. thesis, Trondheim, 1973.

22 J. S. Hoye (unpublished results).

${ }^{23} \mathrm{~J}$. S. Hoye and E. Lomba, J. Chem. Phys. 95, 4502 (1991).

${ }^{24}$ R. Hübner, J. S. Hoye, and K. Olaussen, J. Stat. Phys. 42, 523 (1986).

${ }^{25}$ J. S. Hóye and G. Stell, J. Chem. Phys. 77, 3731 (1982).

${ }^{26} \mathrm{M}$. Winn and G. Kahl, J. Chem. Phys. (to be published). 